\title{
L'Italie ou la genèse du héros romantique
}

Italy and the genesis of the romantic hero

\section{Ronald Perlwitz}

\section{OpenEdition \\ Journals}

Édition électronique

URL : http://journals.openedition.org/cei/1038

DOI : 10.4000/cei. 1038

ISSN : 2260-779X

\section{Éditeur}

UGA Éditions/Université Grenoble Alpes

\section{Édition imprimée}

Date de publication : 31 octobre 2012

Pagination : 211-227

ISBN : 978-2-84310-234-9

ISSN : $1770-9571$

\section{Référence électronique}

Ronald Perlwitz, «L'Italie ou la genèse du héros romantique », Cahiers d'études italiennes [En ligne], 15 2012, mis en ligne le 30 avril 2014, consulté le 26 mars 2021. URL : http://journals.openedition.org/ cei/1038; DOI : https://doi.org/10.4000/cei.1038 


\title{
L'ITALIE OU LA GENÈSE DU HÉROS ROMANTIQUE
}

\author{
Ronald Perlwitz \\ Université Paris-Sorbonne
}

Le 3 septembre 1786 a fait date dans la littérature allemande. À trois heures du matin, muni tout juste d'un sac à dos en fourrure de blaireau et d'un sac de voyage, Johann Wolfgang von Goethe quitte précipitamment la ville thermale de Karlsbad pour se rendre en Italie en passant par la Bavière et le col du Brenner. Dans Le voyage en Italie, son célèbre récit, publié en I8I7, soit plus d'un quart de siècle après son retour en I788, Goethe donne à son départ le caractère d'une fuite. Il aurait voulu s'échapper d'une atmosphère de Weimar devenue trop pesante, laisser derrière lui les tâches astreignantes qui lui incombaient dans l'administration du duché et retrouver la liberté indispensable pour surmonter ce qu'il estimait être une profonde crise artistique. Même au cours des dernières années de sa vie, le Io février I829 par exemple, Goethe parle encore, comme le rapporte son confident Eckermann, d'une «fuite en Italie, afin de retrouver sa productivité poétique ${ }^{\mathrm{I}} »$. Or s'il est vrai que le voyage en Italie marque un tournant important dans son œuvre qui se veut par la suite encore plus résolument tournée vers une esthétique du classique et une conviction profonde de l'autonomie de l'art, il n'en reste pas moins que l'Italie joue, même avant sa découverte physique, un rôle prépondérant dans la pensée goethéenne. Aujourd'hui, nous savons que cette "fuite» n'en était pas vraiment une, qu'une lettre de demande de congés avait été envoyée le 2 septembre à Karl August, duc de Weimar, et que le voyage avait été prévu par Goethe de longue date, même s'il n'en avait pas tenu informé ses proches. La mise en scène autobiographique qui accompagne le voyage montre bien toutefois à quel point la découverte de l'Italie et de Rome en particulier pouvait, pour un auteur allemand du XVIII ${ }^{\mathrm{e}}$ siècle, inspirer un sentiment de renouveau et de rédemption personnelle.

I. "Flucht nach Italien, um sich zu poetischer Produktivität wieder herzustellen." (FA 39, p. 304) 
Longtemps considérée comme le pays de l'épanouissement des sens qu'on visitait pour satisfaire un certain goût de l'exotisme et du pittoresque, l'Italie se transforme au cours du siècle des Lumières, en patrie de l'art et plus précisément de la pureté artistique. De fait, l'italophilie en Allemagne au cours de ces années établit le voyage en Italie comme un véhicule permettant d'atteindre un certain degré de reconnaissance sociale et de culture artistique. On en voudra pour preuve le voyage en Italie qu'entreprend Lessing en 1775 pour accompagner le Prince Leopold de Braunschweig. Quelques années auparavant, en I764, Johann Joachim Winckelmann avait, en publiant son ouvre majeure, Histoire de l'art de l'Antiquité, érigé l'art antique, et plus particulièrement l'art grec, comme idéal artistique absolu, et élevé l'Italie au rang de terre natale des arts que tout artiste, toute personne cultivée se devait de visiter. C'est sur les fondements de cette proclamation de l'art antique comme modèle par excellence de tout travail artistique humain que le classicisme de Weimar va construire son esthétique et c'est aussi dans cette perspective que Goethe perçoit son existence italienne. Dans la couche sociale dont il est issu, l'Italie fait donc partie de la formation des jeunes hommes, qui partent, une fois leurs études terminées, découvrir les richesses de l'antiquité et admirer une nature méridionale à laquelle une multitude d'affects, de comportements et de fantasmes se trouvent rattachés. Son père, Johann Kaspar, avait voyagé en Italie de 1739 à I74I et rédigé à ce sujet un récit en langue italienne dans la meilleure tradition encyclopédique du siècle.

Ne se sentant pas encore prêt, victime de son succès littéraire et social après son départ de Francfort en 1775, Goethe avait juste repoussé l'échéance, sans pour autant jamais abandonner ce qui pour lui revêtait les couleurs d'un rêve d'enfance. À Weimar, son ami Schiller répond à ceux qui s'étonnent de ne pas voir revenir le Conseiller Privé et Ministre, qui ne devait pourtant quitter son poste que quelques semaines pour prendre des vacances : «Depuis l'enfance il avait à coeur de partir en Italie. Son père y était déjà allé2.» Ce n'est donc qu'avec quelques années de retard et avec pour ferme volonté de surmonter sa crise artistique que Goethe se rend en Italie pour y contempler un monde qu'il n'a appris à connaître que dans les livres et qui semble receler la clé de sa vocation d'artiste. Voilà pourquoi il se doit de «fuir». Son voyage n'a pas uniquement une fonction éducative et ne veut plus s'inscrire dans une trajectoire de préparation à une intégration sociale réussie en Allemagne; c’est là précisément que réside

2. "Seine Reise nach Italien hat er von Kindheit an schon im Herzen herumgetragen. Sein Vater war da." Explication de Schiller donnée à Körner (I2.8.1787), citée d'après FA I5/2, p. IO42. 
son originalité. S'il étudie en détail l'œuvre de Winckelmann lors de son séjour, s'il considère comme lui l'art de la Grèce antique comme échelle parfaite de toute production et critique d'art, il n'en reste pas moins que c'est aussi le développement de son individualité, de son originalité de créateur qu'il attend de son expérience italienne. La cour de Weimar lui avait déjà offert toute la reconnaissance sociale et le prestige auxquels un jeune homme d'une trentaine d'années pouvait prétendre à son époque. Restait le rêve artistique... Comme il l'explique dans Poésie et Vérité (I808I83I), l'Italie est pour lui un lieu "parfaitement étranger ${ }^{3}$ " qui incite au retour vers soi justement parce qu'il s'oppose à la patrie allemande où tout lui est quotidien. L'existence mondaine si recherchée et pourtant si pesante est annulée au profit d'une existence de voyageur qui se redéfinit au gré des rencontres. Goethe voyagera sous différents noms d'emprunt et sans aucun serviteur à ses côtés. Il ne veut pas être reconnu et surtout ne veut pas se reconnaître lui-même. "La création de mon propre monde ${ }^{4}$ » devient son objectif principal :

Les journées sont si longues, la réflexion n'est jamais troublée, et les magnifiques images des environs, n'étouffent plus le sens poétique, mais, accompagnées de mouvement et de l'air libre, l'éveillent avec encore plus de vigueur's.

L'expérience italienne aboutira : longtemps Goethe avait pensé pouvoir devenir dessinateur, voire peintre, et l'Italie lui donnera l'occasion de rencontrer quelques-uns des principaux artistes peintres de son époque. À Rome, il partage l'appartement du peintre Johann Heinrich Tischbein qui fera de lui le célèbre portrait Goethe dans la Campagna. À Naples, il se lie d'amitié avec le plus grand paysagiste allemand de son temps, Philipp Hackert qui lui donne non seulement des cours, mais lui apprend aussi à comprendre les paysages méditerranéens et à développer une sensibilité pour la rigueur archaïque et la splendeur des ruines antiques. Guidé par le regard de Hackert, par sa faculté à observer avec précision les moindres détails de la nature et à créer à partir d'eux de véritables portraits de paysages italiens, à la fois réalistes et imaginaires, Goethe perçoit la nature comme entité idéale construite sur les fondements du réel (voir dessin de Hackert : campagne italienne). Si les leçons du peintre lui révèlent finalement la relative faiblesse de son talent de dessinateur, elles le confortent

\footnotetext{
3. "ein ganz Fremdes" (FA I4, p. 304).

4. "Zu meiner Welterschaffung" (FA I5/I, p. I9).

5. "Der Tag ist so lang, das Nachdenken ungestört, und die herrlichen Bilder der Umwelt verdrängen keineswegs den poetischen Sinn, sie rufen ihn vielmehr, von Bewegung und freier Luft begleitet, nur desto schneller hervor." (FA I5/I, p. 25)
} 
cependant dans sa conviction d'écrivain et dans sa confiance en la faculté de l'art à produire, en se fondant sur la nature, une vérité idéale à la fois réaliste et imaginaire. Le classicisme de Hackert couplé à la beauté des paysages italiens donne l'occasion à Goethe de préciser sa conception esthétique et de retrouver sa productivité : le manuscrit d'Iphigénie en Tauride qu'il avait amené avec lui dans ses bagages sera achevé fin I786; il termine également Egmont, travaille à Torquato Tasso et à Faust. Mieux encore : l'Italie ne lui apportera pas seulement une connaissance de soi artistique, mais aussi la découverte du «bien-être ${ }^{6} »$ et de la sensualité. À l'instar de Wilhelm Heinse, auteur dont il avait été proche à l'époque du "Sturm und Drang", l'Italie ne lui apparaît pas uniquement comme terre de jouissance artistique, mais aussi comme un lieu de plaisirs bien plus corporels. Conçu à partir de notes écrites en particulier au cours de plusieurs séjours à Rome (I78I-I783), le roman de Heinse, Ardinghello (I787), avait ajouté à des réflexions détaillées sur l'art l'idée du plaisir comme moteur de toute forme de création et ainsi ravivé le motif de la qualité érotique de l'Italie. Goethe, pour sa part, ne manquera pas de souligner cet aspect qui vient compléter très efficacement sa révolution artistique. Une affaire amoureuse très passionnelle avec une jeune romaine devient ainsi la toile de fond de ses Erotica Romana, les Élégies Romaines (I788-I790), qui reprennent, certes avec un peu plus de correction morale que chez Heinse, le thème de la libération amoureuse comme étape indispensable de l'épanouissement personnel.

La renaissance de Goethe en Italie et la métamorphose de son caractère à la suite de son séjour alimenteront diverses rumeurs à Weimar. Même si Le voyage en Italie, qui correspond à une refonte d'éléments de son journal de voyage, de notes et de lettres, ne paraît que tardivement, l'influence de l'expérience initiatique en Italie devient très vite l'un des sujets majeurs de la scène littéraire allemande et cela bien au-delà des années qui font suite au retour du poète: Goethe va "marquer l'image de l'Italie pour l'ensemble du XIX ${ }^{e}$ siècle ${ }^{7}$ » et sa vision de l'Italie servir de critère dans les débats intellectuels et esthétiques de l'époque. Ainsi, Johann Gottfried Herder, qui part pour Rome en I 788 quelques jours après le retour de Goethe, espère également y trouver une forme de renaissance spirituelle. Sa relation avec Goethe n'étant pas dénuée de tensions et d'un esprit de

6. "Mein eigentlich Wohlleben aber ist in Früchten, in Feigen, auch Birnen, welche da wohl köstlich sein müssen, wo schon Zitronen wachsen.» (FA I5/1, p. 33)

7. "Er [Goethe] hat das Italienbild für das ganze 19. Jahrhundert geprägt, und wer nach Goethe Italien besuchte, kam nicht um die Auseinandersetzung mit seinem Italienbild herum." (G. E. Grimm, U. Breymayer, W. Erhart, "Ein Gefühl vom freierem Leben", Stuttgart, Metzler, I990, p. 2.) 
compétition, il souffre cependant d'évoluer sur un terrain déjà marqué par le regard de son rival. S'il considère les œuvres d'art antiques comme des objets essentiels, détenteurs d'humanité dans sa forme la plus pure, l'Italie lui reste néanmoins une terre étrangère, marquée de clivages sociaux et où l'on vit dans des habitations "épouvantablement sales ${ }^{8}$ ». À Rome, la ville rêvée de Goethe et le centre de son univers artistique, règne d'après Herder l'esprit le plus chaotique. De son voyage, il retiendra surtout l'importance d'ancrer l'art dans un certain concept d'humanisme sublimé qu'il définit aussi par opposition à la déchéance humaine dont il estime avoir été témoin. À cet égard, ses Lettres pour l'avancement de l'humanité (I7931797) sont redevables de son expérience en Italie sur laquelle il s'efforce de poser un regard moins idéalisateur que ne l'avait été celui de Goethe.

Sur la toile de fond de la définition goethéenne de l'Italie se développe ensuite, au cours des dernières années du XVIII ${ }^{\mathrm{e}}$ siècle, l'intérêt de la jeune génération romantique pour l'Italie. L'auteur du roman de formation Les Années d'apprentissage de Wilhelm Meister (I795-I796) fait désormais figure de référence qu'il s'agit à la fois d'imiter et de dépasser. Critiquer, voire dénoncer l'expérience italienne de Goethe fait partie de la pensée d'une génération d'écrivains qui cherche à s'attribuer une nouvelle liberté artistique. En conséquence, la fonction de l'Italie comme étape indispensable de toute formation artistique commence rapidement à évoluer. Or, s'il est courant d'opposer Goethe à la première génération romantique, il l'est beaucoup moins de regarder plus précisément comment cette différence se traduit dans l'approche de l'Italie. Pourtant, peu de critères montrent plus clairement les bouleversements philosophiques, politiques et artistiques en Allemagne au tournant du XIx ${ }^{e}$ siècle que le rapport établi par l'école romantique avec l'Italie. Au tout début du mouvement, dans un texte communément considéré comme l'un des textes fondateurs de la jeune école romantique, Les Épanchements d'un moine ami des arts de Wackenroder et Tieck publié en I796, l'Italie gardait encore à travers la fonction d'exemple suprême qui revient aux dessins et peintures de Raphaël une place centrale dans le développement du nouveau paradigme de la religion de l'art. Juste une année après cependant, en I798, Ludwig Tieck, dont l'apport aux études esthétiques des Épanchements avait été plutôt modeste, publie son second grand roman, Les Pérégrinations de Franz Sternbald et adapte la référence du voyage en Italie au goût romantique. Dorénavant, on ne retrouve plus la construction bien architecturée des romans de formation

8. «Die Häuser der Bürger und gemeinen Leute sehen entsetzlich schmutzig aus» (J. G. Herder, Italienische Reise, p. 233). 
classiques (dont L'Histoire d'Agathon de Wieland, I764, inaugure la tradition). La narration est régulièrement interrompue par des éléments dramatiques, des descriptions de tableaux, des réflexions philosophiques ou des poèmes, ce qui n'empêche pas la trajectoire du peintre Franz Sternbald, qui se déroule au XVI ${ }^{\mathrm{e}}$ siècle, d'aboutir, malgré de nombreux errements, encore et toujours au bonheur retrouvé en Italie. Âgé de 22 ans, le jeune artiste est le disciple favori de son maître Albrecht Dürer, ce qui ne l'empêche pas de quitter la quiétude de sa position pour un voyage d'études qui le mène d'abord aux Pays-Bas, ensuite à Strasbourg, et enfin en Italie du Nord. Au cours de son périple, il rencontre furtivement une jeune fille du nom de Marie, dont le visage lui semble familier. Muni de son portrait, il part à sa recherche, sans négliger pour autant son éducation d'artiste. À Florence, le souvenir de Marie s'estompe quelque peu lorsqu'il succombe aux charmes d'une autre femme. Arrivé à Rome, il retrouve comme par miracle sa bien-aimée et le roman se termine abruptement sur une chanson de Franz, alors qu'une grande partie de la trame du récit n'a pas encore été dénouée ( 46 ans après la première publication du roman, Tieck lui adjoindra l'ébauche d'une seconde partie qu'il n'aura pourtant jamais le temps de rédiger).

Évidemment, pour Tieck, l'influence exercée par le prototype du voyage salvateur en Italie tel que Goethe l'avait vécu reste omniprésente. Malgré son relatif silence à ce sujet ${ }^{9}$, on ne négligera pas non plus la sympathie de Tieck pour Heinse, qui avait également situé au XvI ${ }^{\mathrm{e}}$ siècle son programme de libération de l'ingéniosité et de la passion humaine. La fin fragmentaire des Pérégrinations indique toutefois une évolution à la fois par rapport à l'esthétique classique de Goethe et par rapport à l'utopie de Heinse pour lequel le déplacement vers le passé n'avait pas encore pour fonction de dénoncer la détermination du sujet par son époque. La Renaissance de Heinse était le cadre de l'homme universel, du grand esthète et du grand homme politique qui devait servir d'exemple à l'homme du XviII siècle; selon Tieck en revanche, l'idéalisation du passé n'a plus pour fonction d'offrir une surface de projection à des problèmes contemporains, mais de dévoiler justement le caractère intemporel de la création artistique au-delà de toute contingence culturelle, géographique ou politique.

À y regarder de plus près, on ne peut donc s'empêcher de remarquer les décalages importants entre le parcours de l'artiste romantique et l'imaginaire du voyage en Italie tel qu'il avait été développé quelques années

9. Voir Postface de Max L. Baeumer dans W. Heinse, Ardinghello, p. 657. 
auparavant. Certes, Tieck, comme Goethe avant lui, chante les louanges de l'Italie qui est pour lui le pays du bonheur terrestre et de la sensualité, une terre où règne un renouveau perpétuel et qui accueille chaleureusement l'artiste allemand qui a su s'extraire de sa patrie nordique pour trouver dans le sud l'inspiration d'un nouvel élan créateur : "C'est un peu comme si je respirais avec l'air suave, indolent et pourtant rafraîchissant de l'Italie une autre âme, comme si en mon for intérieur j'engendrais aussi un éternel printemps ${ }^{\mathrm{IO}}$. " Jusqu'ici tout ressemble à l'Italie de Goethe avec ses accents de paradis terrestre. S'il y a donc décalage, il ne se situe pas vraiment dans la perception toujours euphorique de la vie et des paysages italiens, mais plutôt dans le personnage même du héros, ou, pour être plus précis, dans la notion d'héroïsme qui affleure dans le texte de Tieck et qui était absente de l'esthétique d'idéalisation de la nature formulée par Goethe.

Avant d'aller plus avant, il convient d'abord de préciser cette notion qui sous-tend l'ensemble des Pérégrinations de Franz Sternbald. En effet, le voyage romantique n'est plus un voyage vers une meilleure compréhension de soi et, partant, vers un regain de productivité de l'artiste. La théorie romantique va en effet s'attacher à évacuer l'une des notions centrales de la pensée de Goethe, notion très chère au siècle des Lumières : celle de perfectibilité de l'être humain. Ainsi, pour Tieck, qui sans être l'un des premiers penseurs de la génération romantique, subit l'influence de son ami Novalis et de Friedrich Schlegel, la philosophie de l'histoire du siècle des Lumières selon laquelle la libération progressive des sociétés relèverait d'une logique profonde de l'humanité et de sa capacité à évoluer, n'est plus à l'ordre du jour. Elle a été contredite à la fois par la réflexion sur l'art qui s'élabore autour de Friedrich Schlegel ou de Schelling et par la réalité sanglante de la terreur qui suit l'espoir révolutionnaire de 1789. Franz Sternbald, qui en cela se révèle l'incarnation parfaite de l'artiste romantique, formule sa profession de foi artistique alors qu'il n'en est encore qu'au début de son périple. À Anvers, il se voit obligé de justifier son métier d'artiste et le fait en des termes qui placent non seulement l'art sur un terrain jusqu'alors occupé par la tradition religieuse, mais qui soulignent également l'héroïsme emphatique de l'artiste aux prises avec les conventions de la société :

C'est pourquoi j'estime que l'art est le gage de notre immortalité [...]. L'ange qui nous habite aspire à se révéler, mais ne trouve pour cela qu'une énergie humaine; incapable

Iо. "Es ist, als wenn ich mit der weichen, ermattenden und doch erfrischenden Luft Italiens eine andere Seele einzöge, als wenn mein inneres Gemüth auch einen ewigen Frübling hervortriebe.» (TS I6, p. 389) 
de prouver son existence, il agit et nous gouverne de la façon la plus agréable qui soit, pour nous apprendre, comme dans un beau rêve, la foi la plus douce. Ainsi naît, de manière harmonieuse et ordonnée, l'art. Ce que le sage prouve par sa sagesse, ce que le héros confirme par son sacrifice, oui, j'ai le courage de le dire, ce que le martyr atteste par sa mort, le grand peintre l'obtient et l'affirme par ses couleurs ${ }^{\mathrm{II}}$.

Le rôle de l'artiste vient de changer : de formateur, d'architecte de l'humanité, il en est devenu le prophète qui, par sa propre existence, prouve la dignité du substrat d'éternité en chaque être humain. La fonction de création fait ainsi partie de la constitution du sujet; sa capacité à créer relève d'une pratique de soi : "Il ne mène sa vie que pour l'art, et si l'art venait à mourir en lui, il ne saurait plus que faire de la vie qui lui resterait ${ }^{12} . »$ Dans ce contexte, il subit directement le danger d'aliénation qui guette l'artiste dans une société bourgeoise soumise depuis peu aux règles du marché capitaliste (le détour par l'histoire ne trompe pas, c'est bien l'économie de la fin du XviII ${ }^{\mathrm{e}}$ siècle que Tieck vise ici). Franz condamne la position sociale précaire des intellectuels, qui restent soumis aux règles impitoyables de la rémunération, alors que leur unique raison d'être consiste dans le commerce de l'esprit : «Il faut regretter que dans notre vie terrestre l'esprit dépende à ce point de la matière ${ }^{\mathrm{I}}$.»

Il nous faut apporter ici encore quelques précisions : au cours de l'époque romantique, dans le sillage de Kant et surtout dans celui de Fichte, le statut du moi constitué par l'opération transcendantale, commence à changer. L'idéalisme allemand avait maintenu la possibilité d'accéder par les moyens de la connaissance à la condition ontologique de l'existence. En revanche, pour le premier romantisme, la possibilité d'un "savoir absolu» n'est plus donnée, car la caractéristique primordiale de l'absolu demeure, selon lui, dans sa non-perceptibilité. Il lui fallait alors trouver d'autres moyens pour atteindre ce qu'on estimait constituer le fondement unique de l'être humain, le point d'indifférence de la théorie et de la praxis. Novalis, dont les Études sur Fichte (I795-I796), mais aussi dans les Grains de Pollens (I797-I798) et les autres fragments disséminés représentent le système philosophique le plus accompli du premier ro-

II. "So halte ich die Kunst für ein Unterpfand unserer Unsterblichkeit [...]. Der Engel in uns strebt, sich zu offenbaren, und trifft nur Menschenkräfte an, er kann von seinem Daseyn nicht überzengen, und wirkt und regiert nun auf die lieblichste Weise, um uns, wie in einem schönen Traum, den süßen Glauben beizubringen. So entsteht in der Ordnung, in wirkender Harmonie die Kunst. Was der Weise durch Weisheit erhärtet, was der Held durch Aufopferung bewährt, ja, ich bin kühn genug es auszusprechen, was der Märtyrer durch seinen Tod besiegelt, das kann der große Mahler durch seine Farben auswirken und bekräftigen.» (TS 16, p. I66-167)

I2. "Er führt sein Leben nur für die Kunst, und wenn die Kunst ihm abstürbe, würde er nicht wissen, was er mit seinem übrigen Leben beginnen sollte.» (TS I6, p. I67-I68)

13. "Es ist zu bejammern, daß in unserm irdischen Leben der Geist so von der Materie abhängig ist." (TS I6, p. 168) 
mantisme allemand, ouvre alors la voie : toute réflexion qui implique la recherche d'une strate préréflexive de notre être ne peut être opérationnelle qu'à condition de partir d'un constat très simple qui serait celui du vide ouvert en nous par toute tentative d'approximation de l'infini. Or, ce vide ne peut faire l'objet d'un travail de pensée - il lui échappe nécessairement - mais uniquement, à partir du sentiment qu'il suscite, d'une aspiration, d'une quête qui aurait pour objectif de le combler. Arrivé à ce point de son développement philosophique, Novalis a recours à ce qui relève d'un authentique coup de génie intellectuel : étant donné que toute réflexion implique une inversion de perspective, l'opération de recherche du fondement préréflexif de l'humanité ne pourra être conduite que si la "progression apparente du fini vers l'infini» est elle-même réfléchie par une "progression apparente de l'infini vers le fini». Les "résultats de cette réflexion ${ }^{14}{ }^{\star}$ apparaissent donc être les résultats d'une double réflexion, condition indispensable à toute production de l'absolu. La mise en œuvre de ce chassé-croisé intellectuel implique toutefois le fait que le travail de réflexion ne soit pas uniquement cantonné au mode de la conscience, mais se développe aussi à partir de l'endroit où l'activité artistique se substitue au raisonnement philosophique.

Sans entrer plus avant dans le dialogue philosophique qui s'installe entre le premier romantisme allemand et la philosophie idéaliste, il convient néanmoins de souligner ici son influence décisive sur l'œuvre de Tieck. Dans notre optique, évidemment, c'est l'idée de quête qui compte, car elle confère une toute autre dimension au voyage entrepris par le héros romantique. En effet, si on conçoit, comme on l'a fait bien trop souvent, le voyage romantique comme une quête d'absolu entreprise à partir d'une certaine expérience terrestre, on ne s'arrête jamais qu'à mi-chemin de l'opération. Le mouvement de la pensée romantique évolue dans un espace dual et ne saurait fonctionner sans ajouter à toute progression dirigée vers l'infini une progression en sens inverse. Ce que le romantisme postule relève donc d'une autre forme de voyage pour laquelle un voyage intérieur viendra compléter, comme une image dans le miroir de la pensée, le voyage extérieur qu'entreprend le héros. Dans l'un de ses plus célèbres aphorismes, le seizième, de la suite des Grains de pollen (I798), Novalis offre une définition prégnante de sa théorie qui implique de soumettre le voyage à la modalité de la relation de réflexion qui s’installe entre l'extérieur et l'intérieur et inversement :

I4. «Bedürfniß einer Filosofie im Bewußtseyn - Scheinbares Schreiten vom Beschränkten zum Unbeschränkten - Reflexion darüber - Scheinbares Schreiten vom Unbeschränkten zum Beschränkten - Resultate dieser Reflexion — Resultate des Gefühls dieser Reflexion». Extrait des Études sur Fichte (KNA 2, p. II7). 
Le chemin mystérieux va vers l'intérieur. C'est en nous, sinon nulle part, qu'est l'éternité avec ses mondes, le passé et l'avenir. Le monde extérieur est un monde d'ombre, il jette son ombre sur le royaume de lumière. Maintenant, il est vrai, tout nous semble en nous obscurité, chaos informe, solitude; mais comme tout nous paraîtra autre lorsque ces ténèbres se dissiperont et que le corps obscur sera écartét ${ }^{15}$.

Voilà pourquoi il nous fallait poser quelques bases de philosophie romantique. Car on comprend désormais pourquoi le voyage en Italie de Sternbald n'aboutit plus, ne peut plus aboutir à une transformation de l'artiste, comme le voudrait l'exemple du cheminement retracé par Goethe. La nouvelle anthropologie romantique exclut d'emblée ce concept. Pour elle, le vrai voyage se veut un voyage intérieur qui évolue au gré des rencontres et des évènements du voyage extérieur. Pour reprendre Novalis, le voyage dans des contrées étrangères n'est donc jamais que le reflet d'un cheminement intérieur vers les limbes de l'absolu. Partir en Italie devient alors un départ vers soi, qui n'en est pas un, car il exclut d'emblée la notion de retour. C'est ce qui explique la nature fragmentaire du texte de Tieck et confirme aussi le concept de constance du héros en dépit des nouvelles expériences qu'il fait au cours de son odyssée. La femme aimée de Franz est celle dont il porte l'image et même s'il ne résiste pas vraiment toujours aux tentations qui s'offrent à lui, l'union avec Marie — on ne négligera pas la consonance religieuse du nom - demeure le seul objectif possible. À cet égard, l'aboutissement de l'amour à la fin du parcours du jeune peintre représente aussi un aboutissement artistique. Si le voyage intérieur constitue le pendant du voyage extérieur, il n'en reste pas moins que l'opération d'approximation de l'absolu ne peut être déclenchée que par l'intermédiaire concret de l'art. Il est en effet indispensable que le destin de l'artiste se reflète à son tour dans la production artistique pour que la double réflexion puisse fonctionner. De statut de témoignage, le récit de voyage devient, dans la logique romantique, la preuve en soi de l'accessibilité de l'absolu à travers la production artistique. Quoi de plus cohérent alors que d'interrompre le récit au moment où l'amour est atteint? L'union avec la bien-aimée ne marque-t-elle pas symboliquement l'aboutissement de l'aspiration du héros à trouver en lui cette essence infinie qui pouvait le dépasser et le transcender? La «Sehnsucht» du vide, du non-individuel aurait donc trouvé son accomplissement.

15. Traduction d'Albert Béguin, dans Albert Béguin, L'Âme romantique et le rêve, p. 273. "Nach Innen geht der geheimnisvolle Weg. In uns, oder nirgends ist die Ewigkeit mit ihren Welten, die Vergangenheit und Zukunft. Die Außenwelt ist die Schattenwelt, sie wirft ihren Schatten in das Lichtreich. Jetzt scheint es uns freylich innerlich so dunkel, einsam, gestaltlos, aber wie ganz anders wird es uns dünken, wenn diese Verfinsterung vorbey, und der Schattenkörper hinweggerückt ist.» (KNA 2, p. 4I9, $\left.\mathrm{n}^{\circ} \mathrm{I} 6\right)$ 
Mais alors pourquoi avoir perpétué le modèle littéraire du voyage en Italie? Tieck, comme Achim von Arnim, Fouqué, E. T. A. Hoffmann ou Eichendorff, n'a en fait jamais voyagé en Italie et se contente de son imagination. À l'opposé de cette approche, mais à la même époque que les romantiques, en I8or précisément, Johann Gottfried Seume décide, lui, de se rendre à pied en Italie, avec au départ une motivation très humaniste, étant donné qu'il emporte avec lui, malgré leur poids, plusieurs ouvrages classiques d'Homère, en passant par Plaute et Horace jusqu'à Théocrite, dont il rêve de lire les Idylles sur la presqu'île de Syracuse. Toutefois, le récit de voyage qu'il publie en 1803 , Promenade à Syracuse, témoigne moins de sa fascination pour l'Antiquité que de sa volonté de dénoncer les injustices sociales et politiques non seulement en Italie, mais aussi en Europe. Rejetant la prose par trop poétique de son époque, qu'il qualifie de "laitage pour enfants ${ }^{16}$ ", il formule un plaidoyer en faveur d'un récit aux vertus pédagogiques et politiques. L'Italie, idéalisée par Goethe, imaginée par les romantiques, est à ses yeux avant tout un pays où règne «la plus affligeante pauvreté ${ }^{17}$ ", et où des structures féodales, la corruption et l'influence néfaste de la religion ont contribué à cimenter une misère plus que condamnable. Malgré tout ce qui le distingue de l'approche romantique, mot qu'il n'utilise par ailleurs que pour qualifier la beauté de certains paysages ${ }^{18}$, Seume rejoint ses contemporains dans leur revendication d'un art révolutionnaire et d'une littérature qui aurait pour fonction de dessiller les yeux de ses contemporains. Mais là où Seume met l'accent sur la réalité historique, sur les faits navrants qu'il constate lors de son périple, l'école romantique intériorise en quelque sorte l'effort d'éducation et voit en l'Italie un espace d'expérimentation de son approche esthétique et de sa théorie du voyage. Ainsi, pour Novalis, dans son grand roman de formation romantique, Henri d'Ofterdingen (I80o), également resté fragmentaire, le héros n'a même plus besoin de partir dans un pays étranger et peut se contenter de se rendre à Augsbourg. Plus ancré dans la littérature du XVIII ${ }^{\mathrm{e}}$ siècle, Tieck garde volontiers la référence à l'Italie terre des beaux-arts, mais lui ajoute une nouvelle forme de perception plus ambiguë qui s'intéresse plus aux effets du voyage et à l'opération magique de découverte de soi qu’à la fonction éducative de l'Antiquité. L'Italie n'apparaît plus alors comme l'étape indispensable d'une formation

16. "In Romanen hat man uns nun lange genug alte, nicht mehr geleugnete Wabrheiten dichterisch eingekleidet, dargestellt und tausend Mal wiederholt. Ich tadle dieses nicht; es ist der Anfang: aber immer nur Milchspeise für Kinder." (J. G. Seume, Spaziergang, p. I58)

17. "die jämmerlichste Armut» (ibid., p. 247).

I8. "Die Täler am Flusse herunter sind fast alle romantisch schön." (Ibid., p. 206) 
culturelle, artistique et parfois même amoureuse, mais comme un espace à la fois fascinant et contradictoire dans lequel se constitue progressivement la conscience de l'artiste romantique, moderne et fier de l'être.

Dans ce contexte, il n'est pas étonnant de constater que l'Italie commence à perdre pour cette génération son statut de destination exotique privilégiée. L'imaginaire romantique se tourne également vers d'autres pays, se construit de nouveaux paradis terrestres. Ainsi, Friedrich Schlegel, principale instance théorique du premier romantisme, élabore dans son Voyage en France (1803) une philosophie de l'histoire de l'Europe, qui repose sur une téléologie résolument tournée vers l'Orient. Selon lui, l'Europe serait désormais scindée en deux, en une Europe du Sud et une Europe du Nord, c'est-à-dire en deux ensembles dont les caractéristiques reproduisent la «séparation européenne du classique et du romantique ${ }^{19}$ ». Incapable de reconnaître les liens qui lient poésie et philosophie, arts et sciences, l'Europe dans la pensée schlegelienne apparait condamnée à ne jamais recouvrer son unité par ses propres moyens. Sa réunification présupposant une nouvelle conscience religieuse, Schlegel conseille maintenant à ceux qui voudraient construire une nouvelle Europe de partir en Inde afin d'y apprendre la religion "comme on part en Italie pour apprendre l'art ${ }^{20} »$. S'il est donc encore possible d'apprendre les techniques de production artistique en Italie, Schlegel n'y projette plus l'espoir d'une humanité meilleure. Bien au contraire : l'Italie fait désormais partie intégrante de cette Europe en proie à des bouleversements révolutionnaires que les intellectuels allemands regardent souvent avec la plus grande méfiance.

Reste à élucider la problématique de l'héroïsme romantique déjà sousjacente dans le roman de Tieck. En effet, au cours des Pérégrinations de Franz Sternbald l'étoffe du héros reposait sur sa capacité à s'extraire du monde et à préserver sa dignité d'artiste en dépit du matérialisme ambiant. Avec l'arrivée de la deuxième génération romantique, cette dimension existentielle de la biographie de l'artiste romantique va définitivement gagner le devant de la scène. Et on ne s'étonnera pas de constater dans ce contexte le rôle déterminant qui revient une nouvelle fois à l'Italie. Reprenant le fil de la sacralité de l'art, la nouvelle génération trouve dorénavant en Italie le terrain idéal pour une mise en scène du conflit entre sensualité et spiritualité, mais aussi entre orthodoxie chrétienne et esthétique prophétique. De matrice d'une renaissance artistique, l'Italie devient le pays où le héros

19. "jene europäische Trennung des Klassischen und des Romantischen" (KSA 7, p. 74).

20. "man möchte demjenigen, der Religion sehen will, raten, er solle, wie man nach Italien geht um die Kunst zu lernen, eben so zu seinem Zwecke nach Indien reisen" (KSA 7, p. 74). 
romantique doit plus que jamais prouver son statut d'élu. Ainsi, dans l'œuvre d'E. T. A. Hoffmann, l'Italie prend tantôt un aspect menaçant, tantôt un aspect parfaitement quotidien. On en voudra pour preuve le récit d'Ignaz Denner (I8I4) issu des Contes nocturnes, dans lequel le docteur Trabbachio fait régner la terreur à Naples et en Allemagne en se livrant à de sanglantes opérations alchimiques. Dans la Cour d'Arthur (I8I5), le lecteur retrouve certes le modèle du voyage en Italie et du jeune homme accueilli à Rome par une colonie d'artistes allemands au sein de laquelle son talent de peintre commence à s'imposer. Et comme Sternbald, le jeune Traugott part effectivement en Italie à la recherche d'une bien-aimée idéalisée. Mais l'expérience italienne a des accents de trivialité et n'ouvre plus dans l'art hoffmannien de perspective d'échange avec l'infini. Felizitas, la femme rêvée, lui échappe tout d'abord par mégarde et ensuite parce qu'elle choisit une existence bourgeoise de mère de famille. Elle se désincarne alors en «rêve délicieux ${ }^{2 \mathrm{2I}}$ » qui l'inspire dans son art, mais sans justifier pour autant un vrai processus d'introspection. L'Italie démystifiée est un lieu de refuge pour l'artiste qui y trouve, en la personne d'une jeune Italienne avec laquelle il se marie, une existence stable à son goût. À l'infinitude du voyage romantique, Hoffmann substitue la possibilité très concrète d'une vie d'artiste en harmonie avec la société. Une vision à laquelle l'éloignement de l'Italie, comme pour Heinse, donne une touche utopique.

Mais si le héros romantique éprouve la nostalgie de sa réintégration dans la société, il n'en reste pas moins qu'en ce début de XIX ${ }^{\mathrm{e}}$ siècle la réalité sociale évolue dans une direction qui lui rend cette possibilité de plus en plus improbable. Le paradigme de l'autonomie de l'art associé à la conviction de sa suprématie a encore contribué à creuser le fossé déjà présent au siècle des Lumières entre l'idéal moral formulé par l'art et la réalité politique et sociale. Confronté à cet abyme, l'artiste romantique hésite et abandonne progressivement la position très exposée de prophète. À la recherche d'harmonie, il découvre progressivement les dissonances qui accompagnent son existence d'artiste et toutes les conditions extérieures qui l'aliènent. C'est là-dessus que se fonde l'approche polémique de Joseph von Eichendorff qui en I8I8 publie l'un des premiers contes résolument modernes de la littérature allemande : La statue de marbre. Eichendorff connaît bien, très bien, les dangers qu'implique une recherche artistique de convergences conduite au nom d'une unité interne indéfinissable. Son

2I. "sie gestaltete sich im Innern, wie ein wonnevoller Traum, dessen duftiger Schimmer sein ganzes Leben umflo $\beta_{\text {" }}\left(\mathrm{HW}_{3}\right.$, p. 164). 
héros, Florio, évolue à cet effet dans un espace qui ressemble, même si aucune précision historique n'est apportée, à l'Italie de la Renaissance. La ville de Lucques offre l'arrière-plan de l'histoire du jeune homme qui tombe éperdument amoureux d'une femme mystérieuse, dont l'apparition magique sur les bords d'un lac évoque la Naissance de Vénus de Botticelli. La description de la ville toutefois s'estompe et disparait lorsque surgissent les visions oniriques et les scènes irréelles qui se déroulent dans le palais rêvé de Vénus. Envoûté par la beauté et l'ivresse qui émanent d'elle, Florio méprise de plus en plus le monde réel et, solitaire, s'adonne à ses rêveries. Lorsque la femme adorée lui apparait sous forme de statue, il manque de perdre la raison. Torturé par sa passion, il se résout enfin à quitter Lucques et rencontre devant les portes de la ville son ami Fortunato qui lui explique qu'à l'endroit où il s'imaginait dans un opulent palais magique s'élèvent les ruines d'un ancien temple de Vénus. Fortunato entonne alors un chant sacré qui conjure le pouvoir néfaste de la déesse antique, ce qui permet à Florio, libéré du sort qui l'avait aveuglé, de retrouver une jeune fille, Bianka, à laquelle il avait été sensible avant de subir l'emprise des visions suggérées par la déesse : "Je viens de renaître, j'ai l'impression que tout ira bien maintenant que je vous ai retrouvée ${ }^{22}$ ", avoue-t-il à la jeune fille qui, heureuse de voir l'homme qu'elle admirait lui jurer fidélité, prend l'allure d'un ange ${ }^{23}$. Aussitôt la campagne italienne s'éclaire et le temple sinistre disparaît à l'horizon.

Malgré le happy end qu'Eichendorff veut encore donner à son histoire, l'ambivalence de la représentation de l'Italie ne manque pas d'éveiller l'attention. Évidemment, il s'agit d'un conte. Et si le héros parvient finalement à surmonter la tentation qu'incarne Vénus, c'est parce qu'il trouve le droit chemin de la religion. Or, l'évolution de l'ensemble du mouvement romantique le prouve : un des dangers de la sacralisation de l'art, de l'art rédempteur, c'est le retour de la tradition qu'il voulait dépasser, le retour des vieux prêtres de la religion. L'art qui se voulait autonome, qui voulait ériger sa propre église vient, en dépit de tout le travail intellectuel accompli, de se remettre au service de l'ancienne. Eichendorff en est conscient et offre une fin aussi rayonnante que trompeuse. Le moins que l'on puisse dire, c'est qu'il ne se montre pas vraiment persuadé d'avoir trouvé la bonne solution. Son Italie est à l'image de cette incertitude; elle

22. "Ich bin wie neu geboren, es ist mir, als würde noch alles gut werden, seit ich euch wiedergefunden.» (EW 2, p. 428)

23. "Bianka blickte ihn, statt aller Antwort selber wie fragend, mit ungewisser, noch halb zurückgehaltener Freude an und sah recht wie ein heiteres Engelsbild auf dem tiefblauen Grunde des Morgenhimmels aus. " (EW 2, p. 428) 
est à l'image des hésitations, des illusions et des angoisses qui traversent son texte. C'est une Italie contradictoire qui est à la fois le lieu privilégié du christianisme et, vu depuis l'Allemagne, la terre où les mythes antiques perdurent. C'est un pays prédisposé à accueillir un conte qui hésite entre époque ancienne et monde contemporain, le lieu privilégié des ambiguïtés, des ruptures et des conflits qu'implique la conception romantique de l'art aux prises avec un infini qui lui échappe et un fini qu'il ne peut manquer de critiquer. Quant au héros, il porte déjà en lui la conscience aristocratique qui sera celle de l'artiste moderne, chez Baudelaire, chez Heine ou chez Wagner dont le Tannhäuser continue l'histoire de Florio. Car la particularité du sujet romantique se constitue à travers les liens que son imaginaire tisse avec le monde et corrélativement à travers le rapport entre fini et infini qu'il parvient à établir. Au cours de la première époque romantique, ce sujet avait encore l'avantage de se savoir soutenu par un système philosophique clairement structuré. Mais Eichendorff se place volontairement à l'écart de ce système qu'il accuse d'exercer la fascination dangereuse d'une fausse religion. Il l'accuse de vouloir envoûter ses proies comme la musique de l'attrapeur de rats de Hamelin. La substance intemporelle des mythes n'est à ses yeux qu'une illusion, et la téléologie une erreur de pensée que la réalité ne cesse d'invalider. Laissé pour compte, le sujet s'en remet à la religion, sans grande conviction et non sans avoir subi auparavant toutes les passions et s'être adonné à toutes les superstitions. L'héroïsme, au sens où Eichendorff l'entend, se trouve là, dans la faculté du sujet à supporter la confrontation avec la réalité et à défendre néanmoins une conception artistique aux prises avec la possibilité de l'éternel. Même au cours de la seconde époque romantique l'Italie reste donc une école. Elle était le miroir du voyage romantique, elle se transforme désormais en école, voire en laboratoire de la tentation. Ce n'est plus la perfection de ses œuvres d'art qui forme l'artiste allemand, mais sa confrontation avec ces «colonnes magnifiques à moitié ensevelies» recouvertes «d'une nature sauvage fleurie et luxuriante». Près de l'étang, dans lequel elle se reflète, gît une «statue de marbre en partie détruite ${ }^{24} »$. C'est en l'observant, en l'imaginant, en la reconstruisant que naît l'artiste. Et Goethe, malgré toute sa méfiance à l'égard d'une révolution romantique de l'art, aurait été d'accord. Au moins sur ce point.

24. "schöne halb in die Erde versunkene Säulen und künstlich gehauene Steine, alles von einer üppig blühenden Wildnis [...] überdeckt” (EW 2, p. 423). 


\section{Bibliographie}

\section{Sources}

Eichendorff Joseph von, Werke, vol. 2 : Ahnung und Gegenwart, Erzählungen I, éd. Wolfgang Frühwald, Brigitte Schillbach et Hartwig Schultz, Francfort-sur-le-Main, Deutscher Klassiker Verlag, 1985. (EW 2) Goethe Johann Wolfgang, Sämtliche Werke, Briefe, Tagebücher und Gespräche, 40 vol., éd. Friedmar Apel, Hendrik Birus et suiv., Francfortsur-le-Main, Deutscher Klassiker Verlag, 1985-1999. (FA I-40)

HeInse Wilhelm, Ardinghello und die glückseligen Inseln, Stuttgart, Reclam, 1998.

Herder Johann Gottfried, Italienische Reise. Briefe und Tagebuchaufzeichnungen 1788-I789, éd. Albert Meier et Heide Hollmer, Munich, DTV, 1988.

Hoffmann E. T. A., Werke in nicht-nummerierten Einzelbänden, 5 vol., éd. Walter Müller-Seidel et Friedrich Schnapp, Munich, Winkler, I960-198I. (HW I-5)

Novalis, Schriften. Die Werke Friedrich von Hardenbergs, 7 vol., éd. Paul Kluckhohn, Richard Samuel, Hans-Joachim Mähl et Gerhard Schulz, Darmstadt, Stuttgart, Wissenschaftliche Buchgesellschaft, I968-I988. (KNA I-7)

Schlegel Friedrich, Kritische Ausgabe seiner Werke, 34 vol., éd. Ernst Behler, Munich, Paderborn, Vienne, Zurich, 1958 sq. (KSA I-34)

Seume Johann Gottfried, Mein Leben, Spaziergang nach Syrakus im Jahre I802, Mein Sommer I805, éd. Jörg Drews et Sabine Kyora, dans Johann Gottfried Seume, Werke, Francfort-sur-le-Main, Deutscher Klassiker Verlag, 1993.

Tieck Ludwig, Schriften, 28 vol., Berlin, Reimer, I828-I854, réimpr. Berlin, De Gruyter, 1966. (TS I-28)

\section{Littérature secondaire}

Auerochs Bernd, Die Entstehung der Kunstreligion, Göttingen, Vandenhoeck \& Ruprecht, 2009.

BÉGuIn Albert, L'Âme romantique et le rêve, Paris, José Corti, I99I.

Frank Manfred, Der kommende Gott. Vorlesungen über die neue Mythologie, Francfort-sur-le-Main, Suhrkamp Wissenschaft, 1982.

—, "Unendliche Annäherung": Die Anfänge der philosophischen Frühromantik, Francfort-sur-le-Main, Suhrkamp Wissenschaft, 1997.

Götting Roland, E. T. A. Hoffmann und Italien, Francfort-sur-le-Main, Lang, 1992. 
Grimm Gunter E., Breymayer Ursula et Erhart Walter, "Ein Gefühl vom freierem Leben". Deutsche Dichter in Italien, Stuttgart, Metzler, I990.

Hausmann Frank-Rutger (dir.), "Italien in Germanien«: deutsche ItalienRezeption von I750-I850, Akten des Symposiums der Stiftung Weimarer Klassik, Herzogin-Anna-Amalia-Bibliothek, Schiller-Museum, Tübingen, Narr, 1996.

Kremer Detlef, Romantik, Stuttgart, Metzler Verlag, 2006.

Schanze Helmut, Romantik-Handbuch, Tübingen, Kröner, 1994.

Schmitz-Emans, Einführung in die Literatur der Romantik, Darmstadt, Wissenschaftliche Buchgesellschaft, 2004.

Schultz Hartwig, Joseph von Eichendorff, Francfort-sur-le-Main, Leipzig, Insel, 2007.

Tortarolo Edoardo, Diesseits und Jenseits der Alpen: deutsche und italienische Kultur im I8. Jahrhundert, traduction Ludger Scherer, Leipzig, Leipziger Univ.-Verl., 20II.

Uerlings Herbert, Friedrich von Hardenberg, genannt Novalis. Werk und Forschung, Stuttgart, Metzler Verlag, I99I.

Witte Bernd, Buck Theo, Dahnke Hans-Dietrich, Otto Regine et Scнмidt Peter (éd.), Goethe-Handbuch, 5 vol., Stuttgart, Metzler Verlag, I996-I998. 